\title{
Erratum to: Elastic properties of six silicate garnet end members from accurate ab initio simulations
}

\author{
Alessandro Erba • Agnes Mahmoud • \\ Roberto Orlando $\cdot$ Roberto Dovesi
}

Published online: 29 November 2013

(C) Springer-Verlag Berlin Heidelberg 2013

\section{Erratum to: Phys Chem Minerals}

DOI 10.1007/s00269-013-0630-4

Unfortunately, during the typesetting process the first digits of some values in Table 1 have been truncated. The correct version of Table 1 is given below.

The online version of the original article can be found under doi:10.1007/s00269-013-0630-4.

A. Erba $(\square) \cdot$ A. Mahmoud $\cdot$ R. Orlando $\cdot$ R. Dovesi Dipartimento di Chimica and Centre of Excellence NIS

(Nanostructured Interfaces and Surfaces), Università di Torino,

via Giuria 5, 10125 Torino, Italy

e-mail: alessandro.erba@unito.it 
Table 1 Elastic constants $C_{v u}(\mathrm{GPa})$ and adiabatic bulk modulus $K_{S}(\mathrm{GPa})$ of the six silicate garnet end members

\begin{tabular}{|c|c|c|c|c|c|c|}
\hline & References & Technique & $C_{11}$ & $C_{12}$ & $C_{44}$ & $K_{S}$ \\
\hline \multirow[t]{11}{*}{ Pyr } & Isaak et al. (1976) & Ultrasonic, at RTP & 287 & 105 & 92 & 166 \\
\hline & Bonczar et al. (1977) & Ultrasonic, at RTP & 292 & 106 & 92 & 168 \\
\hline & Leitner et al. (1980) & Brillouin scattering, at RTP & 295 & 117 & 90 & 177 \\
\hline & O’Neill et al. (1989) & Brillouin scattering, at RTP & 296 & 111 & 92 & 173 \\
\hline & O’Neill et al. (1991) & Brillouin scattering, at RTP & 298 & 110 & 93 & 172 \\
\hline & Sinogeikin et al. (2000) & Brillouin scattering, at RTP & 297 & 108 & 93 & 171 \\
\hline & Lu et al. (2013) & Brillouin scattering, at RTP & 291 & 107 & 92 & 168 \\
\hline & Pavese (1999) & Empirical potentials & 298 & 113 & 93 & 174 \\
\hline & Winkler et al. (1999) & Semiempirical potentials & 339 & 132 & 115 & 201 \\
\hline & Mittal et al. (2001) & Semiempirical potentials & 314 & 116 & 91 & 182 \\
\hline & This study & Ab initio B3LYP & 296 & 109 & 89 & 171 \\
\hline \multirow[t]{4}{*}{ Alm } & Isaak et al. (1976) & Ultrasonic, at RTP & 310 & 115 & 93 & 180 \\
\hline & O’Neill et al. (1989) & Brillouin scattering, at RTP & 309 & 111 & 96 & 177 \\
\hline & Mittal et al. (2001) & Semiempirical potentials & 318 & 113 & 90 & 182 \\
\hline & This study & $\mathrm{Ab}$ initio B3LYP & 306 & 108 & 88 & 174 \\
\hline \multirow[t]{4}{*}{ Spe } & Isaak et al. (1976) & Ultrasonic, at RTP & 302 & 108 & 96 & 172 \\
\hline & Bass $(1989)^{\mathrm{a}}$ & Brillouin scattering, at RTP & 310 & 114 & 95 & 179 \\
\hline & Mittal et al. (2001) & Semiempirical potentials & 313 & 109 & 89 & 177 \\
\hline & This study & Ab initio B3LYP & 310 & 114 & 94 & 179 \\
\hline \multirow[t]{8}{*}{ Gro } & Halleck (1973) & Ultrasonic, at RTP & 318 & 98 & 101 & 171 \\
\hline & Isaak et al. (1976) & Ultrasonic, at RTP & 320 & 96 & 102 & 171 \\
\hline & Bass $(1989)^{\mathrm{b}}$ & Brillouin scattering, at RTP & 322 & 91 & 105 & 168 \\
\hline & Winkler et al. (1999) & Semiempirical potentials & 397 & 98 & 94 & 198 \\
\hline & Mittal et al. (2001) & Semiempirical potentials & 305 & 103 & 84 & 170 \\
\hline & Kawai et al. (2012) & $\mathrm{Ab}$ initio LDA & 334 & 98 & 104 & 177 \\
\hline & This study & Ab initio B3LYP & 323 & 95 & 105 & 171 \\
\hline & Bass (1986) & Brillouin scattering, at RTP & 289 & 92 & 85 & 157 \\
\hline \multirow[t]{2}{*}{ And } & Jiang et al. (2004) & Brillouin scattering, at RTP & 284 & 88 & 83 & 155 \\
\hline & This study & Ab initio B3LYP & 281 & 88 & 83 & 152 \\
\hline \multirow[t]{2}{*}{ Uva } & Bass (1986) & Brillouin scattering, at RTP & 304 & 91 & 84 & 162 \\
\hline & This study & Ab initio B3LYP & 306 & 83 & 87 & 157 \\
\hline
\end{tabular}

Present computed values are compared with the previously measured experimental and simulated data. Few details about the techniques used in each cited work are given (RTP stands for room temperature and pressure)

a Extrapolated from a $95 \%$ Spessartine sample

b Extrapolated from a $99 \%$ Grossular sample 